\title{
BOUNDS FOR DIOPHANTINE QUINTUPLES
}

\author{
Minai Cipu and Yasutsugu Fujita \\ IMAR, Romania and Nihon University, Japan
}

\begin{abstract}
A set of $m$ positive integers $\left\{a_{1}, \ldots, a_{m}\right\}$ is called a Diophantine $m$-tuple if the product of any two elements in the set increased by one is a perfect square. The conjecture according to which there does not exist a Diophantine quintuple is still open. In this paper, we show that if $\{a, b, c, d, e\}$ is a Diophantine quintuple with $a<b<c<d<e$, then $b>3 a$; moreover, $b>\max \left\{21 a, 2 a^{3 / 2}\right\}$ in case $c>a+b+2 \sqrt{a b+1}$.
\end{abstract}

\section{INTRODUCTION}

A set of $m$ positive integers $\left\{a_{1}, \ldots, a_{m}\right\}$ with the property that the product of any two elements in the set increased by one is a perfect square is called a Diophantine $m$-tuple.

The first example $\{1,3,8,120\}$ of a Diophantine quadruple was found by Fermat. In general, for a given Diophantine triple $\{a, b, c\}$, the set $\left\{a, b, c, d_{+}\right\}$ is always a Diophantine quadruple, where

$$
d_{+}=a+b+c+2 a b c+2 \sqrt{(a b+1)(a c+1)(b c+1)}
$$

(see [1]). A set of the type $\left\{a, b, c, d_{+}\right\}$is called a regular Diophantine quadruple. Fermat's set $\{1,3,8,120\}$ is regular, and, according to [17], in [18] and independently in [1] it is conjectured that any Diophantine quadruple is regular, implying the folklore conjecture that there does not exist a Diophantine quintuple. In [8] Dujella obtained results very close to settling this conjecture by proving that there does not exist a Diophantine sextuple and that there exist only finitely many Diophantine quintuples. Later bounds on the number of Diophantine quintuples are provided in $[9,11,16]$. Historical and recent

2010 Mathematics Subject Classification. 11D09, 11B37, 11J68.

Key words and phrases. Diophantine $m$-tuples, Pell equations, hypergeometric method. 
developments of the study of Diophantine m-tuples are found on Dujella's webpage: http://web.math.pmf.unizg.hr/ duje/dtuples.html.

The existence of Diophantine sets boils down to solving systems of generalized Pell equations. Recently, the first author ([6, Lemma 2.4]; see also Lemma 3.4 below) improved the lower bound for hypothetical solutions to the system relevant in the study of Diophantine quintuples (note that a similar assertion is obtained in [23]). Using this lemma, he investigated the properties that Diophantine quintuples should have, and in particular updated the known upper bounds for the fourth element $d$ ([6, Theorem 2.1]) and for the number of Diophantine quintuples ([6, Theorem 1.3]).

The aim of this paper is to show the non-existence of Diophantine quintuples such that the two smallest elements are rather close to each other. More precisely, we prove the following.

TheOREM 1.1. There exists no Diophantine quintuple $\{a, b, c, d, e\}$ with $a<b<c<d<e$ and $b \leq 3 a$.

Theorem 1.2. There exists no Diophantine quintuple $\{a, b, c, d, e\}$ with $a<b<c<d<e, c>a+b+2 \sqrt{a b+1}$ and $b \leq \max \left\{21 a, 2 a^{3 / 2}\right\}$.

In addition to the above mentioned result [6, Lemma 2.4], a key role in our deliberations plays an amelioration (Theorem 2.2 in Section 2) of Rickert's theorem (see [21]; cf. also [5, Theorem 3.2], [12, Theorem 2.5]). The improvements were obtained by adjusting an idea found in Bennett's paper [3] to our situation. The proofs of Theorems 1.1 and 1.2 are given in the last section.

\section{A VERSION OF RICKERT'S THEOREM}

For any irregular quadruple $\{a, b, c, d\}$ with $a<b<c<d$, a lower bound for the second element $b$ has been obtained by using a version ([10, Lemma $5])$ of the Baker-Davenport reduction method ([2, Lemma $])$ in the proof of [13, Theorem 1.2]. Computations performed in order to establish the above mentioned theorem (and described at length on the last page of [13]) can be summarized as follows.

Lemma 2.1. Suppose that $\{a, b, c, d\}$ is a Diophantine quadruple with $a<$ $b<c<d_{+}<d$.

- If $b<2 a$, then $b>21000$.

- If $2 a \leq b \leq 8 a$, then $b>130000$.

- If $b>8 a$, then $b>2000$.

As already mentioned, the existence of Diophantine sets hinges on the solvability of a certain system of generalized Pell equations. It is also well known that solutions to such systems appear as common terms to several second-order linear recurrent sequences. In order to get a better upper bound 
for the index of appearance of a hypothetical solution in the relevant sequence, we slightly improve [12, Theorem 2.5], which is a version of Rickert's theorem $([21])$.

THEOREM 2.2. Let $a, b$ and $N$ be integers with $0<a \leq b-5, b>2000$ and $N \geq 3.706 a^{\prime} b^{2}(b-a)^{2}$, where $a^{\prime}=\max \{b-a, a\}$. Assume that $N$ is divisible by $a b$. Then the numbers $\theta_{1}=\sqrt{1+b / N}$ and $\theta_{2}=\sqrt{1+a / N}$ satisfy

$$
\max \left\{\left|\theta_{1}-\frac{p_{1}}{q}\right|,\left|\theta_{2}-\frac{p_{2}}{q}\right|\right\}>\left(\frac{1.413 \cdot 10^{28} a^{\prime} b N}{a}\right)^{-1} q^{-\lambda}
$$

for all integers $p_{1}, p_{2}, q$ with $q>0$, where

$$
\lambda=1+\frac{\log \left(10 a^{-1} a^{\prime} b N\right)}{\log \left(2.699 a^{-1} b^{-1}(b-a)^{-2} N^{2}\right)}<2 .
$$

The proof of the original result, as well as of all subsequent versions of it, relies on a very general construction recalled below.

Lemma 2.3. ([5, Lemma 3.1]) Let $\theta_{1}, \ldots, \theta_{m}$ be arbitrary real numbers and $\theta_{0}=1$. Assume that there exist positive real numbers $l, p, L$ and $P$ with $L>1$ such that for each positive integer $k$, we can find integers $p_{i j k}$ $(0 \leq i, j \leq m)$ with nonzero determinant,

$$
\left|p_{i j k}\right| \leq p P^{k} \quad(0 \leq i, j \leq m)
$$

and

Then

$$
\left|\sum_{j=0}^{m} p_{i j k} \theta_{j}\right| \leq l L^{-k} \quad(0 \leq i \leq m)
$$

$$
\max \left\{\left|\theta_{1}-\frac{p_{1}}{q}\right|, \ldots,\left|\theta_{m}-\frac{p_{m}}{q}\right|\right\}>c q^{-\lambda}
$$

holds for all integers $p_{1}, \ldots, p_{m}, q$ with $q>0$, where

$$
\lambda=1+\frac{\log P}{\log L} \quad \text { and } \quad c^{-1}=2 \operatorname{mpP}(\max \{1,2 l\})^{\lambda-1} .
$$

Proof of Theorem 2.2. We apply Lemma 2.3 with $m=2$ and $\theta_{1}, \theta_{2}$ as in Theorem 2.2. For $0 \leq i, j \leq 2$ and arbitrary integers $a_{i}$ (which will be specialized to $0, a$ and $b$ in due time), let $p_{i j}(x)$ be the polynomial defined by

$$
p_{i j}(x)=\sum_{i j}\left(\begin{array}{c}
k+\frac{1}{2} \\
h_{j}
\end{array}\right)\left(1+a_{j} x\right)^{k-h_{j}} x^{h_{j}} \prod_{l \neq j}\left(\begin{array}{c}
-k_{i l} \\
h_{l}
\end{array}\right)\left(a_{j}-a_{l}\right)^{-k_{i l}-h_{l}}
$$

where $k_{i l}=k+\delta_{i l}$ with $\delta_{i l}$ the Kronecker delta, $\sum_{i j}$ denotes the sum over all non-negative integers $h_{0}, h_{1}, h_{2}$ satisfying $h_{0}+h_{1}+h_{2}=k_{i j}-1$, and $\prod_{l \neq j}$ denotes the product from $l=0$ to $l=2$ omitting $l=j$ (which is the 
expression (3.7) in [21] with $\nu=1 / 2)$. As seen in the proof of $[12$, Theorem $2.5]$, we have

$$
f(x):=2^{2 k-1} \prod_{l \neq j}\left(a_{j}-a_{l}\right)^{k_{i l}+h_{l}} p_{i j}(x) \in \mathbb{Z}[x] .
$$

Noting

$$
\left(\begin{array}{c}
-k_{i l} \\
h_{l}
\end{array}\right)=(-1)^{h_{l}}\left(\begin{array}{c}
k_{i l}+h_{l}-1 \\
h_{l}
\end{array}\right),
$$

we see from [4, Lemma 4.1] (or [3, Lemma 3.2]) that $P_{2}(k)$ divides the greatest common divisor, denoted by $\Pi_{2}(k)$, of the coefficients of $f(x)$, where $P_{2}(k)$ is the product over all primes $p$ satisfying $p>\sqrt{2 k+1}, \operatorname{gcd}(p, 2 k)=1$ and $\{(k-1) / p\}>3 / 4$ (as usual, $\{t\}$ denotes the fractional part of a real number $t$ ); in particular, we have $\Pi_{2}(k) \geq P_{2}(k)$. Denoting by $J_{l}(k)$ the open interval $((k-1) / l, 4(k-1) /(4 l-1))$, we obtain

$$
P_{2}(k) \geq \prod_{l=1}^{\left[\frac{k-1}{\sqrt{2 k-1}}\right]} \prod_{\substack{p \in J_{l}(k) \\ \operatorname{gcd}(p, 2 k)=1}} p .
$$

Now we appeal to Corollary $2^{*}$ of Theorem $7^{*}$ (the case $(c, d)=(15,70877)$ in the table) and the Note added in proof from [22] in order to get a lower bound for the right-hand side of (2.1). If $k \geq 903683$, then

$$
\begin{aligned}
\sum_{l=1}^{13} \sum_{\substack{p \in J_{l}(k) \\
\operatorname{gcd}(p, 2 k)=1}} \log p & >\sum_{l=1}^{13}\left(\frac{4}{4 l-1}-\frac{4}{15(4 l-1) \log \frac{4(k-1)}{4 l-1}}-\frac{1.000081}{l}\right)(k-1) \\
& -\log k \\
> & 0.47064(k-1)-\log k>0.4706 k,
\end{aligned}
$$

which implies that $\Pi_{2}(k)>e^{0.4706 k}>1.6^{k}$. For each $1 \leq k \leq 903682$, put

$$
g(k)=\sum_{l=1}^{\left[\frac{k-1}{\sqrt{2 k+1}}\right]} \sum_{\substack{p \in J_{l}(k) \\ \operatorname{gcd}(p, 2 k)=1}} \log p-k \log 1.6 .
$$

Then, with the help of a computer we find that $g(k)>-31.342$, where the minimal value of $g(k)$ is attained in the case of $k=607$. This estimate yields

$$
\Pi_{2}(k)>\frac{1.6^{k}}{e^{31.342}}>\frac{1.6^{k}}{4.09 \cdot 10^{13}},
$$

which also holds for $k \geq 903683$.

If we take $a_{0}=0, a_{1}=a$ and $a_{2}=b$, the proof of [12, Theorem 2.5] shows that

$$
p_{i j k}:=2^{-1}\left[4 a b(b-a)^{2} N\right]^{k} \Pi_{2}(k)^{-1} p_{i j}(1 / N) \in \mathbb{Z},
$$


which together with (2.2) and the proof of [15, Theorem 21] implies that

$$
\left|p_{i j k}\right|<p P^{k}, \quad\left|\sum_{j=0}^{2} p_{i j k} \theta_{j}\right|<l L^{-k},
$$

where

$$
\begin{aligned}
p & =\frac{4.09 \cdot 10^{13}}{2}\left(1+\frac{a^{\prime}}{2 N}\right)^{1 / 2}<2.046 \cdot 10^{13}, \\
P & =\frac{32\left(1+\frac{3 b-a}{2 N}\right) a b(b-a)^{2} N}{1.6 \zeta}<\frac{10 a^{\prime} b N}{a} \\
(\zeta & =\left\{\begin{array}{ll}
a^{2}(2 b-a) & \text { if } b-a \geq a, \\
(b-a)^{2}(a+b) & \text { if } b-a<a
\end{array}\right), \\
l & =\frac{4.09 \cdot 10^{13}}{2} \cdot \frac{27}{64}\left(1-\frac{b}{N}\right)^{-1}<8.628 \cdot 10^{12}, \\
L & =\frac{1.6}{4 a b(b-a)^{2} N} \cdot \frac{27}{4}\left(1-\frac{b}{N}\right)^{2} N^{3}>\frac{2.699 N^{2}}{a b(b-a)^{2}} .
\end{aligned}
$$

Moreover, the assumption $N \geq 3.706 a^{\prime} b^{2}(b-a)^{2}$ implies that

$$
\lambda=1+\frac{\log \left(10 a^{-1} a^{\prime} b N\right)}{\log \left(2.699 a^{-1} b^{-1}(b-a)^{-2} N^{2}\right)}<2
$$

and

$$
\begin{aligned}
c^{-1} & <4 \cdot 2.046 \cdot 10^{13} \cdot \frac{10 a^{\prime} b N}{a}\left(17.256 \cdot 10^{12}\right)^{\lambda-1} \\
& <\frac{1.413 \cdot 10^{28} a^{\prime} b N}{a} .
\end{aligned}
$$

This completes the proof of Theorem 2.2.

\section{Proofs of the main Results}

Let $\{a, b, c\}$ be a Diophantine triple with $a<b<c$ and $r, s, t$ the positive integers satisfying $a b+1=r^{2}, a c+1=s^{2}, b c+1=t^{2}$. Assume that $\{a, b, c, d\}$ is a Diophantine quadruple. Then, there exist positive integers $x, y, z$ satisfying $a d+1=x^{2}, b d+1=y^{2}, c d+1=z^{2}$. Eliminating $d$ from these equations, we obtain the following system of generalized Pell equations

$$
\begin{aligned}
& a z^{2}-c x^{2}=a-c, \\
& b z^{2}-c y^{2}=b-c .
\end{aligned}
$$


The solutions of equations (3.1) and (3.2) can be respectively expressed as $z=v_{m}$ and $z=w_{n}$ with positive integers $m$ and $n$, where

$$
\begin{aligned}
& v_{0}=z_{0}, \quad v_{1}=s z_{0}+c x_{0}, \quad v_{m+2}=2 s v_{m+1}-v_{m}, \\
& w_{0}=z_{1}, \quad w_{1}=t z_{1}+c y_{1}, \quad w_{n+2}=2 t w_{n+1}-w_{n} .
\end{aligned}
$$

Lemma 3.1. (cf. [7, Lemma 12]) Let $N=a b c$ and let $\theta_{1}, \theta_{2}$ be as in Theorem 2.2. Then all positive solutions of the system of Diophantine equations (3.1) and (3.2) satisfy

$$
\max \left\{\left|\theta_{1}-\frac{s b x}{a b z}\right|,\left|\theta_{2}-\frac{t a y}{a b z}\right|\right\}<\frac{c}{2 a} z^{-2} .
$$

Lemma 3.2. Suppose that there exist integers $m \geq 3$ and $n \geq 2$ such that $z=v_{2 m}=w_{2 n}$ and $\left|z_{0}\right|=1$, and that $c \geq 3.706 a^{\prime} b(b-a)^{2} / a$. Then, $\log z>n \log (4 b c)$.

Proof. One can prove this lemma in the same way as [7, Lemma 25] (see also the proof of [12, Lemma 2.10]).

Now we are ready to obtain an upper bound for the solution.

LEMma 3.3. Suppose that there exist integers $m \geq 3$ and $n \geq 2$ such that $z=v_{2 m}=w_{2 n}$ and $\left|z_{0}\right|=1$, and that $c \geq 3.706 a^{\prime} b(b-a)^{2} / a$. Then,

$$
n<\frac{4 \log \left(8.406 \cdot 10^{13} a^{1 / 2}\left(a^{\prime}\right)^{1 / 2} b^{2} c\right) \log \left(1.643 a^{1 / 2} b^{1 / 2}(b-a)^{-1} c\right)}{\log (4 b c) \log \left(0.2699 a\left(a^{\prime}\right)^{-1} b^{-1}(b-a)^{-2} c\right)} .
$$

Proof. By Lemma 2.1, we may apply Theorem 2.2 with $q=a b z, p_{1}=$ $s b x, p_{2}=t a y$, and $N=a b c$. In view of Lemma 3.1, we have

$$
z^{2-\lambda}<0.7065 \cdot 10^{28} a a^{\prime} b^{4} c^{2}<\left(8.406 \cdot 10^{13} a^{1 / 2}\left(a^{\prime}\right)^{1 / 2} b^{2} c\right)^{2} .
$$

From

$$
\frac{1}{2-\lambda}=\frac{\log \left(2.699 a b(b-a)^{-2} c^{2}\right)}{\log \frac{2.699 a(b-a)^{-2} c}{10 a^{\prime} b}}<\frac{2 \log \left(1.643 a^{1 / 2} b^{1 / 2}(b-a)^{-1} c\right)}{\log \left(0.2699 a\left(a^{\prime}\right)^{-1} b^{-1}(b-a)^{-2} c\right)}
$$

and Lemma 3.2 we obtain the asserted inequality.

We need a recent improvement on the lower bound for the solution. Its proof is similar to the proof of [23, Lemma 2].

Lemma 3.4. ([6, Lemma 2.4]) If there exist integers $m \geq 3$ and $n \geq 2$ such that $z=v_{2 m}=w_{2 n}$ and $\left|z_{0}\right|=1$, then $m>0.5 b^{-1 / 2} c^{1 / 2}$.

The following is useful to refine some estimates for the solutions.

LEMMA 3.5. If $\{a, b\}$ is a Diophantine pair with $b>a+2$, then

$$
b-a \geq 2 \sqrt{a+1}+1 .
$$


Proof. The assumption $b-a>2$ implies $a+b-2 r \geq 1$, from which the assertion can be easily deduced.

Proof OF Theorem 1.1. Assuming the contrary, we will show a contradiction. First of all, we note that if we replace " $c$ " in (3.1), (3.2), (3.3), (3.4) by " $d$ ", then $z=v_{2 m}=w_{2 n}$ and $\left|z_{0}\right|=1$ hold for some $m \geq 3$ and $n \geq 2$ (see the part just before the proof of [12, Theorem 2.1]).

Suppose first that $b<2 a$. Then $a^{\prime}=a$ and $b-a<b / 2$. Since

$$
\begin{gathered}
\frac{3.706 a^{\prime} b(b-a)^{2}}{a}<3.706 b \cdot \frac{b^{2}}{4}=0.9265 b^{3}, \\
d>4 a b c>4 \cdot \frac{b}{2} \cdot b(a+b+2 r)>2\left(\frac{1}{2}+1+\sqrt{2}\right) b^{3}>5.8284 b^{3},
\end{gathered}
$$

we can apply Lemma 3.2 with $c$ replaced by $d$. By Lemma 3.5 (which can be applied thanks to the main result in [14]) we have

$$
a^{1 / 2}(b-a)^{-1} \leq \frac{\sqrt{a}}{2 \sqrt{a+1}+1}<0.5,
$$

which together with Lemma 3.3 implies

$$
n<\frac{4 \log \left(8.406 \cdot 10^{13} b^{3} d\right) \log \left(0.8215 b^{1 / 2} d\right)}{\log (4 b d) \log \left(1.0796 b^{-3} d\right)} .
$$

Since the right-hand side is a decreasing function of $d$ and $d>5.8284 b^{3}$, we have

$$
n<\frac{4 \log \left(4.9 \cdot 10^{14} b^{6}\right) \log \left(4.789 b^{7 / 2}\right)}{\log \left(23.3136 b^{4}\right) \log (6.292)}<h_{1}(b),
$$

where

$$
h_{1}(b)=\frac{21 \log (280.78 b) \log (1.565 b)}{\log (2.197 b) \log (6.292)} .
$$

On the other hand, we know by Lemma 3.4 with $m \leq 2 n$ that

$$
n>0.25 b^{-1 / 2} d^{1 / 2}>0.6035 b,
$$

which implies $0.6035 b<h_{1}(b)$. Therefore we obtain $b<200$, which contradicts Lemma 2.1.

Suppose secondly that $2 a \leq b \leq 3 a$, so that $a^{\prime}=b-a \leq 2 b / 3$. Since

$$
\begin{gathered}
\frac{3.706 a^{\prime} b(b-a)^{2}}{a}=3.706 \cdot \frac{b}{a} \cdot(b-a)^{3}<3.295 b^{3}, \\
d>4 a b c \geq 4 \cdot \frac{b}{3} \cdot b(a+b+2 r)>3.317 b^{3},
\end{gathered}
$$


we can apply Lemma 3.2 with $d$ instead of $c$. Since $a^{\prime}=b-a \leq 2 b / 3$, and $a^{1 / 2}(b-a)^{1 / 2} \leq b / 2$ by the arithmetic mean-geometric mean inequality, we see from Lemma 3.3 that

$$
n<\frac{4 \log \left(4.203 \cdot 10^{13} b^{3} d\right) \log (2.3236 d)}{\log (4 b d) \log \left(0.3036 b^{-3} d\right)} .
$$

In a similar way to the above, we have $d>3.317 b^{3}$ and $n<h_{2}(b)$, where

$$
h_{2}(b)=\frac{18 \log (227.712 b) \log (1.976 b)}{\log (1.908 b) \log (1.007)},
$$

which together with $n>0.4553 b$ shows that $0.4553 b<h_{2}(b)$. Therefore we obtain $b<97000$, which contradicts Lemma 2.1. This completes the proof of Theorem 1.1.

Proof of Theorem 1.2. Once again, we argue by reduction to absurd. By [19, Lemma 4], if $c>a+b+2 r$ then actually $c>4 a b$. Having in view Theorem 1.1, we may also assume that $b>3 a$. Hence, $a^{\prime}=b-a$.

First suppose $b \leq 21 a$. Then we find

$$
\begin{aligned}
\frac{3.706 a^{\prime} b(b-a)^{2}}{a} & =3.706 \cdot \frac{b}{a} \cdot(b-a)^{3}<67.23 b^{3}, \\
d & >4 a b c>16 a^{2} b^{2} \geq 0.0362 b^{4} .
\end{aligned}
$$

Since $b>2000$ according to Lemma 2.1, the hypothesis of Lemma 3.3 is fulfilled.

From $3 a<b$ it results $a^{1 / 2} b^{1 / 2}(b-a)^{-1}<\sqrt{3} / 2$, while the upper bound on $b$ in terms of $a$ entails $a b^{-1}(b-a)^{-3}>21^{2} b^{-3} / 20^{3}$. Using these inequalities, Lemma 3.3 yields

$$
n<\frac{4 \log \left(4.203 \cdot 10^{13} b^{3} d\right) \log (1.4229 d)}{\log (4 b d) \log \left(67.2123^{-1} b^{-3} d\right)} .
$$

As the right side of the previous relation is a function decreasing in $d$, for $d>0.0362 b^{4}$ one obtains

$$
n<\frac{22.4 \log (54.996 b) \log (0.4764 b)}{\log (0.6794 b) \log \left(1856.7^{-1} b\right)} .
$$

This upper bound on $n$ is compatible with the lower bound $n>b^{3 / 2} / 21$ derived with Lemma 3.4 only for $b<1980$. Thus, Lemma 2.1 leads us to a contradiction.

Suppose secondly $b \leq 2 a^{3 / 2}$. Since

$$
\begin{aligned}
\frac{3.706 a^{\prime} b(b-a)^{2}}{a} & <\frac{3.706 b^{4}}{(b / 2)^{2 / 3}}<5.883 b^{10 / 3}, \\
d & >16(b / 2)^{4 / 3} b^{2}>6.349 b^{10 / 3},
\end{aligned}
$$


we may apply Lemma 3.3, and thereby obtain

$$
n<\frac{4 \log \left(4.203 \cdot 10^{13} b^{3} d\right) \log (1.4229 d)}{\log (4 b d) \log \left(0.17002 b^{-10 / 3} d\right)} .
$$

Similarly to the first case, $d>6.349 b^{10 / 3}$ implies

$$
n<\frac{760 \log (189.597 b) \log (1.936 b)}{39 \log (2.109 b) \log (1.0794)} .
$$

Comparing this upper bound with $n>2^{-2 / 3} b^{7 / 6}$ deduced from Lemma 3.4, we obtain $b<1490$. As this range is prohibited by Lemma 2.1, the proof of Theorem 1.2 is complete.

At the request of the referee, we close with a few remarks on the uniqueness of the extension of a Diophantine triple $\{a, b, c\}(a<b<c)$ with $a$ and $b$ very close to each other, such as $b \leq 3 a$ or $b \leq \max \left\{21 a, 2 a^{3 / 2}\right\}$, where we proved the non-extendibility to a Diophantine quintuple.

The main technical obstacle which presently prevents to obtain such a result is the non-availability of a lower bound on indices $m$ and $n$ for which $v_{m}=w_{n}$ as powerful as that given in Lemma 3.4 and which should be valid for all combinations odd-even. The best lower bounds proved so far for $m n$ odd are of order $b^{-3 / 4} c^{1 / 4}$ (see $[6,13]$ ). Clearly, such a bound is irrelevant in our problem unless $c \approx b^{\alpha}$ with $\alpha$ sensibly greater than 3 . Moreover, a small gap between $b$ and $c$ does not even allow one to show that the indices $m$ and $n$ have the same parity.

ACKNowledgements.

The second author was partially supported by JSPS KAKENHI Grant Number 25400025. Both authors are grateful to an anonymous referee for suggestions included in the final version of the paper.

\section{REFERENCES}

[1] J. Arkin, V. E. Hoggatt and E. G. Strauss, On Euler's solution of a problem of Diophantus, Fibonacci Quart. 17 (1979), 333-339.

[2] A. Baker and H. Davenport, The equations $3 x^{2}-2=y^{2}$ and $8 x^{2}-7=z^{2}$, Quart. J. Math. Oxford Ser. (2) 20 (1969), 129-137.

[3] M. A. Bennett, Simultaneous approximation to pairs of algebraic numbers, in: CMS Conf. Proc. 15 (1995), 55-65.

[4] M. A. Bennett, Simultaneous rational approximation to binomial functions, Trans. Amer. Math. Soc. 348 (1996), 1717-1738.

[5] M. A. Bennett, On the number of solutions of simultaneous Pell equations, J. Reine Angew. Math. 498 (1998), 173-199.

[6] M. Cipu, Further remarks on Diophantine quintuples, Acta Arith. 168 (2015), 201219.

[7] A. Dujella, An absolute bound for the size of Diophantine $m$-tuples, J. Number Theory 89 (2001), 126-150.

[8] A. Dujella, There are only finitely many Diophantine quintuples, J. Reine Angew. Math. 566 (2004), 183-214. 
[9] A. Dujella, On the number of Diophantine m-tuples, Ramanujan J. Math. 15 (2008), $37-46$.

[10] A. Dujella and A. Pethő, A generalization of a theorem of Baker and Davenport, Q. J. Math. 49 (1998), 291-306.

[11] C. Elsholtz, A. Filipin and Y. Fujita, On Diophantine quintuples and D(-1)quadruples, Monatsh. Math. 175 (2014) 227-239.

[12] A. Filipin and Y. Fujita, The number of Diophantine quintuples II, Publ. Math. Debrecen 82 (2013), 293-308.

[13] A. Filipin, Y. Fujita, and A. Togbé, The extendibility of Diophantine pairs I: the general case, Glas. Mat. Ser. III 49 (2014), 25-36.

[14] Y. Fujita, The extensibility of Diophantine pairs $\{k-1, k+1\}$, J. Number Theory 128 (2008), 322-353.

[15] Y. Fujita, Any Diophantine quintuple contains a regular Diophantine quadruple, J. Number Theory 129 (2009), 1678-1697.

[16] Y. Fujita, The number of Diophantine quintuples, Glas. Mat. Ser. III 45 (2010), 15-29.

[17] P. E. Gibbs, Computer Bulletin 17 (1978), 16.

[18] P. E. Gibbs, Some rational Diophantine sextuples, Glas. Mat. Ser. III 41 (2006), 195-203.

[19] B. W. Jones, A second variation on a problem of Diophantus and Davenport, Fibonacci Quart. 16 (1978), 155-165.

[20] The PARI Group, PARI/GP, version 2.3.5, Bordeaux, 2010, available from http: //pari.math.u-bordeaux.fr/.

[21] J. H. Rickert, Simultaneous rational approximations and related Diophantine equations, Math. Proc. Cambridge Philos. Soc. 113 (1993), 461-472.

[22] L. Schoenfeld, Sharper bounds for the Chebyshev functions $\theta(x)$ and $\psi(x)$ II, Math. Comp. 30 (1976), 337-360.

[23] W. Wu and B. He, On Diophantine quintuple conjecture, Proc. Japan Acad. Ser. A Math. Sci. 90 (2014), 84-86.

\section{Cipu}

Simion Stoilow Institute of Mathematics of the Romanian Academy

Research unit nr. 5

P.O. Box 1-764, RO-014700 Bucharest

Romania

E-mail: Mihai.Cipu@imar.ro

Y. Fujita

Department of Mathematics

College of Industrial Technology

Nihon University

2-11-1 Shin-ei, Narashino, Chiba

Japan

E-mail: fujita.yasutsugu@nihon-u.ac.jp

Received: 21.3.2014.

Revised: 5.6.2014. 